\title{
Embolism of the popliteal artery after anterior cruciate ligament reconstruction: a case report and literature review
}

\author{
R. P. A. Janssen $\cdot$ H. A. G. M. Sala
}

Received: 4 April 2007/ Accepted: 4 May 2007/Published online: 20 June 2007

(C) Springer-Verlag 2007

\begin{abstract}
Arterial complications after anterior cruciate ligament reconstruction (ACLR) are rare. We present a case report of a 44-year-old male patient with a subtotal occlusion of the popliteal artery, with sensory loss in the foot, 17 days after ACLR. Embolectomy and anticoagulant therapy led to full recovery of the peripheral arterial circulation. The sensory loss of the foot also fully recovered. To our knowledge, this is the first case report of an embolus of the popliteal artery after ACLR without relation to graft fixation. A literature review on vascular complications after ACLR is presented.
\end{abstract}

Keywords Embolism · Popliteal artery · Vascular complication - Anterior cruciate ligament reconstruction . Embolectomy $\cdot$ Literature review

\section{Introduction}

Vascular complications after anterior cruciate ligament reconstruction (ACLR) are rare. Few peer reviewed case reports have been reported with various techniques of reconstruction [1, 3-9]. Allum did not report vascular lesions as a complication after ACLR in a review article on this subject [2]. The origin of vascular lesions after ACLR may be venous or arterial [4]. We present a case of arterial

R. P. A. Janssen ( $₫)$

Department of Orthopaedic Surgery and Traumatology,

Máxima Medical Center, PO Box 7777,

5500 MB Veldhoven, The Netherlands

e-mail: R.Janssen@mmc.nl

H. A. G. M. Sala

Kliniek ViaSana, PO Box 4, 5450 AA Mill, The Netherlands embolism of the popliteal artery after ACLR. A literature review on vascular complications after ACLR is presented.

\section{Case report}

A 44-year-old male has a previous medical history of open medial and lateral ligament repair of the right knee 15 years previously (motor accident). Functional instability of the right knee due to ACL deficiency was the reason for referral to our service. There were no signs of posterior nor posterolateral instability. An ACLR was performed with a quadruple hamstring graft. The graft was fixed with a Bone Mulch Screw on the femoral side and a WasherLoc device in the tibia (Arthrotek, Inc. Warsaw, USA). The latter is a spiked washer with bicortical screw fixation. Total tourniquet time was $90 \mathrm{~min}$. No complications were noted during or after surgery. Thromboprophylaxis for deep venous thrombosis was given by means of Low Molecular Weight Heparin (2500 IE daily) during hospital stay. The hospital recovery was uneventful.

On the 17th day of post surgery, he experienced pain and swelling in the popliteal fossa of the right knee. The complaints partially resolved with physiotherapy. Two days later, the fossa pain returned with alterations of skin color, sensory loss and an increasing cold right foot. He was referred to a vascular surgeon. Adequate dorsal pedal and posterior tibial pulses were noted. Duplex ultrasound examination showed no sign of venous thrombosis. Angiography revealed a subtotal occlusion of the popliteal artery at the level of the superior genicular artery (Fig. 1). An embolectomy was performed using a Fogarty catheter inserted in the femoral artery. The pedal pulses were diminished after embolectomy and a second angiography was performed. The occlusion at the level of the popliteal artery 


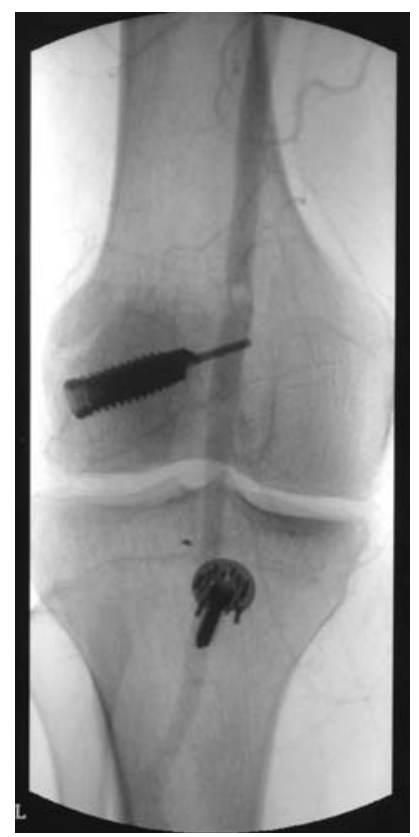

Fig. 1 Angiography of the right knee showing subtotal occlusion of the popliteal artery at the level of the superior genicular artery (reproduced with permission from [4])

was no longer detected. No further emboli were noted, however, the peripheral flow qualified as too slow and suspect of small distal occlusions. Anticoagulant therapy with intravenous heparin as well as epidural analgesia was administered until complete recovery of peripheral circulation. The patient developed a superficial infection of the groin wound, treated by antibiotics. He was mobilized and discharged after 8 days. Sensory loss of the foot slowly recovered after 4 months. Vascular analysis in rest and strenuous activity was performed at 4 months. He had no more complaints, symmetrical ankle-brachial index in both legs and intact pulses at the foot and ankle. Vascular analysis did not reveal any other possible cause for arterial emboli. The patient has full range of motion of the right knee with a Lachman and anterior drawer test of 0-2 $\mathrm{mm}$ (International Knee Documentation Committee) and absent pivot shift test.

\section{Discussion}

Vascular complications after ACLR are rare. The origin of vascular lesions may be venous or arterial [4]. A case of fatal pulmonary embolism after ACLR with venous origin has been published recently [5]. Hypothesis of the cause was a hereditary coagulopathy. Arterial lesions of the popliteal artery after ACLR have been presented in few peer reviewed case reports even with an all-inside technique of arthroscopic ACL reconstruction and fixation as well as any type of graft [4].
Roth et al. [8] described an occlusion of the proximal popliteal artery. A composite graft consisting of a polypropylene ligament augmentation and the middle third of the quadriceps-patellar tendon was fixed to the lateral femur with a staple. The artery was trapped between the graft and the femur. A saphenous bypass was performed 6 weeks post surgery.

Spalding et al. [9] reported a case of unilateral claudication 8 years after ACL reconstruction with use of a GoreTex ligament. A cyst had formed around the femoral insertion of the ruptured synthetic ligament and was excised without vascular repair.

Evans et al. [3] reported a pseudoaneurysm of the medial inferior genicular artery following ACL reconstruction using a central third patellar tendon graft fixed with interference screws. At 5 weeks, ligation of the artery and removal of the thrombus led to full recovery. The cause of the lesion was elevation of the periosteum on the medial side of the tibia for tibial tunnel preparation.

Aldridge et al. [1] described an avulsion of the middle genicular artery after a bone-patellar tendon-bone autograft fixed with interference screws. Surgical exploration at 4 weeks revealed a tear in the popliteal artery. There was no rupture of the posterior capsule. Probable cause for the avulsion of the middle genicular artery was the debridement of the femoral ACL remnant tissue.

We have previously published two cases of popliteal artery lesions caused by a drill for bicortical tibia fixation after quadruple hamstring ACLR [6, 7]. In the first case, the drill had caused an intimal lesion at the level of the infragenicular popliteal artery which led to the pseudoaneurysm. Vascular repair was performed 12 days after ACL reconstruction but sensory loss of the saphenous and medial plantar nerves was still present at 4 months followup [7]. The second case was a simultaneous traumatic pseudoaneurysm and thrombosis of the popliteal artery after ACLR. At surgical exploration, the thrombosis was in line with the drill hole for bicortical tibial fixation. There was no apparent relation of the femoral fixation device to the pseudoaneurysm of the supragenicular popliteal artery. This pseudoaneurysm was thought to be pre-existent. The pseudoaneurysm was ligated and a venous jump graft was performed to bypass the thrombosis located more distally in the popliteal artery [6].

In this review of the literature on arterial complications after ACLR, all cases are associated with direct damage to the popliteal artery at time of ACLR [1, 3, 6-9]. There was no apparent direct damage to the popliteal artery in the 44year-old male patient presented in this case report. The popliteal artery occlusion was not in line with either the femoral nor tibial fixation device. Vascular analysis did not reveal any pre-existent vascular causes for arterial embolus formation proximal to the popliteal artery. Our hypothesis 
of the cause was the traumatic knee dislocation 15 years previously. Precursors could have been pre-existent intimal vascular damage or adhesions of the artery at the level of the superior genicular artery in combination with the use of the tourniquet and ACLR.

\section{Conclusion}

Awareness of possible arterial complications after ACL reconstruction is essential for early diagnosis. Clinical symptoms of pain in the popliteal fossa and sensory deficits in lower leg and foot should prompt the physician to analyze possible injuries of the popliteal artery. The differential diagnosis should include compartment syndrome and deep venous thrombosis. Doppler examination as well as intact pedal arterial pulses are unreliable in diagnosing arterial lesions after ACL reconstruction. Contrast-, CTor MRI-angiography are the diagnostic tools of choice. Immediate surgical exploration is indicated to limit limb ischemia and neurological damage [4].

Acknowledgments Parts of this case report will be published in the book: Prodromos CC (ed) The anterior cruciate ligament: reconstruction and basic science. Copyright 2007 by Saunders an imprint of Elsevier (in press).

Conflict of interest None of the authors have any conflict of interest.

\section{References}

1. Aldridge JM III, Weaver JP, Mallon WJ (2002) Avulsion of the middle genicular artery: a previously unreported complication of anterior cruciate ligament repair. A case report. Am J Sports Med 30(5):748-750

2. Allum R (2003) Aspects of current management. Complications of arthroscopic reconstruction of the anterior cruciate ligament. J Bone Joint Surg (Br) 85:12-16

3. Evans JD, Boer de MT, Mayor P, Rees D, Guy AJ (2000) Pseudoaneurysm of the medial inferior genicular artery following anterior cruciate ligament reconstruction. Ann R Coll Surg Engl 82:182-184

4. Janssen RPA (2007) Vascular complications after anterior cruciate ligament reconstruction. In: Prodromos CC (ed) The anterior cruciate ligament: reconstruction and basic science. Copyright 2007 by Saunders an imprint of Elsevier (in press)

5. Janssen RPA, Sala HAGM (2007) Fatal pulmonary embolism after anterior cruciate ligament reconstruction. Am J Sports Med 35(6): 1000-1002

6. Janssen RPA, Sala HAGM, Prakken WJ (2007) Simultaneous traumatic pseudoaneurysm and thrombosis of the popliteal artery after anterior cruciate ligament reconstruction. Inj Extra. doi:10.1016/j.injury.2007.01.038

7. Janssen RPA, Scheltinga MRM, Sala HAGM (2004) Pseudoaneurysm of the popliteal artery after anterior cruciate ligament reconstruction with bicortical tibial screw fixation. Arthroscopy 20(1):E4-E6

8. Roth JH, Bray RC (1988) Popliteal artery injury during anterior cruciate ligament reconstruction: brief report. J Bone Joint Surg (Br) 70:840

9. Spalding TJW, Botsford DJ, Ford M, Marks PH (1996) Popliteal artery compression: a complication of Gore-tex anterior cruciate ligament reconstruction. J Bone Joint Surg (Br) 78:151-152 